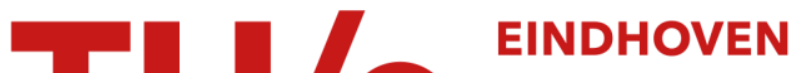 \\ UNIVERSITY OF \\ TECHNOLOGY
}

\section{Smart MVILV transformer for future grids}

Citation for published version (APA):

Kadurek, P., Cobben, J. F. G., \& Kling, W. L. (2010). Smart MV/LV transformer for future grids. In Proceedings of the 2010 International Symposium on Power Electronics Electrical Drives Automation and Motion (SPEEDAM), 14-16 June 2010, Pisa, Italy (pp. 1700-1705). Institute of Electrical and Electronics Engineers. https://doi.org/10.1109/SPEEDAM.2010.5545067

DOI:

10.1109/SPEEDAM.2010.5545067

Document status and date:

Published: 01/01/2010

\section{Document Version:}

Publisher's PDF, also known as Version of Record (includes final page, issue and volume numbers)

\section{Please check the document version of this publication:}

- A submitted manuscript is the version of the article upon submission and before peer-review. There can be important differences between the submitted version and the official published version of record. People interested in the research are advised to contact the author for the final version of the publication, or visit the $\mathrm{DOI}$ to the publisher's website.

- The final author version and the galley proof are versions of the publication after peer review.

- The final published version features the final layout of the paper including the volume, issue and page numbers.

Link to publication

\section{General rights}

Copyright and moral rights for the publications made accessible in the public portal are retained by the authors and/or other copyright owners and it is a condition of accessing publications that users recognise and abide by the legal requirements associated with these rights.

- Users may download and print one copy of any publication from the public portal for the purpose of private study or research.

- You may not further distribute the material or use it for any profit-making activity or commercial gain

- You may freely distribute the URL identifying the publication in the public portal.

If the publication is distributed under the terms of Article 25fa of the Dutch Copyright Act, indicated by the "Taverne" license above, please follow below link for the End User Agreement:

www.tue.nl/taverne

Take down policy

If you believe that this document breaches copyright please contact us at:

openaccess@tue.nl

providing details and we will investigate your claim. 


\title{
Smart MV/LV transformer for future grids
}

\author{
P. Kadurek*, J. F. G. Cobben **, and W. L. Kling * \\ * Eindhoven University of Technology, Eindhoven, (The Netherlands) \\ ** Alliander and Eindhoven University of Technology, Arnhem and Eindhoven, (The Netherlands)
}

\begin{abstract}
An increasing amount of distributed generation in the future grid will be connected at the customer's point of connection. The generation within the low voltage grid will affect the power quality of customers connected to this grid. Hence, the concept of a Smart MV/LV transformer as a part of the Smart MV/LV substation is discussed in this paper. The main aims are to support the accommodation of distributed generation among the $L V$ grid and to ensure that the voltage levels will comply with the power quality standards for all customers connected to that grid.
\end{abstract}

Index Terms--distributed generation, power system modeling, smart metering, voltage level control.

\section{INTRODUCTION}

Nowadays, electricity is the backbone of our society and industry. Electricity consumption has been steadily increasing over the last decades and continuous growth is expected for the years to come. The reliability and quality of this electricity supply are inherent needs of our lives. The design and commissioning of the electricity grids is a long term task. Today's grids were designed some decades ago and most of them will be in operation for following decades. Every change of the grid's structure involves a huge amount of administration, planning and monetary resources. Therefore, future developments should be assessed with a suitable approach and today's grids should be as a steady part. Hence, newly designed grids should be able to handle and cooperate with the future components and customer's appliances for upcoming several decades. It will be not an easy task for the grid planners, because the traditional supply paradigm is going to change in the near future. The present (more or less) unidirectional power flows are going to change and will become more complex. One of the challenges in the future electricity grid will be the increasing penetration of renewable resources connected directly at the customer's point of connection (POC).

Due to the grid's design, the medium voltage (MV) to low voltage (LV) substation is an interesting spot, where it is possible to take some action to improve the power quality (PQ) of the customers' voltage. The PQ could be improved or has to be maintained to comply with the standards for supply voltage. In recent years, the supply voltage standards have increased the requirement on the supplied voltage and a further tightening of the standards

This research has been performed within the framework of the IOP-EMVT research program „Intelligent Power Systems“, which is financially supported by SenterNovem. SenterNovem is an agency of the Dutch Ministry of Economic Affairs. will be expected in the years to come. One of the most important requirements for customers is the voltage level at their POC. Today's substations do include nor the PQ monitoring or devices to take the action in order to improve the PQ at the customer's POC [1], [2].

The future grids should be familiar with the concept of the distributed generation (DG) which will take place near the consumption. Among others, the DG concept leads to a decrease of the losses of the networks, the possibly to handle the increasing demand with current grid structures and an increase of the independency of involved parts of the networks. On the contrary, the increasing penetration of DG could have side effects on the PQ for the customers. One of the fundamental premises for future grid with DG generation is the need to keep the voltages at the POC conformable to the PQ standards. Also, the power flows within the grid should be within the limits given by the network constraints [3], [4], [5].

The traditional design of the MV/LV substation consists of an $\mathrm{MV} / \mathrm{LV}$ transformer, where the tap adjustment is possible only as an off-line operation. The concept of a controllable (smart) transformer, as a part of the intelligent substation, will allow the on-line control of the voltage on the LV side of the transformer [6].

Therefore, different strategies for the voltage control of the smart transformer's tap position and their impact on the voltages at the POCs are discussed in this paper.

The limitations of present day's grids with regard to the accommodation of DG are pointed out (section II). The maximum amount of DGs connected to the standard grid configuration is presented (section III). Afterwards, the concept of intelligent substation and smart transformer is described (section IV) and the different control strategies for the smart transformer are discussed (section V). The experimental results are presented (section VI) and the recommendations for implementation and data infrastructure are discussed (VII).

\section{CURRENT GRID AND ACCOMmODATION OF DG}

Nowadays, the MV/LV transformer has a fixed tap position, mutually offsetting the voltage drop among the LV and MV grid. Due to a high or low load, voltages among the grid vary. According to the simulation, the worst case scenarios are a combination of minimum load and considerable amount of DGs dispatched, or the combination of a maximum load and a minimum of DGs dispatched. 


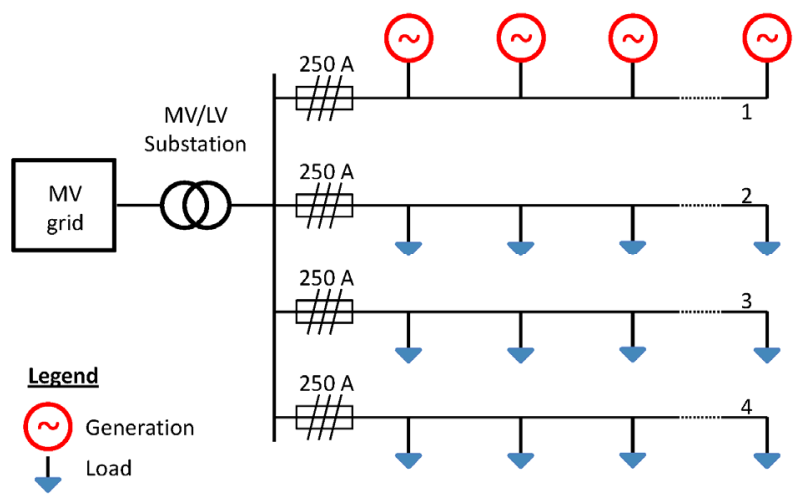

Fig. 1. Model of a typical Dutch LV grid with assumed DGs concentrated among one feeder.

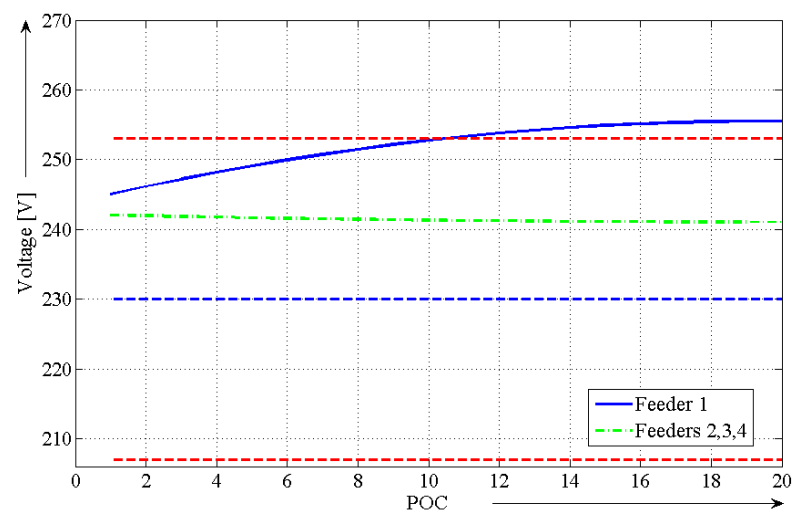

Fig. 2. Voltages at the POCs connected to different feeders with and without DG dispatched. The voltage limits $\left( \pm 10 \% U_{n}\right)$ are presented as red dashed lines.

In order to demonstrate the impact of the DGs dispatched to the LV grid, we assume a grid situation of the LV grid with aggregated DGs in one feeder, see Fig. 1. A considerable amount of DGs is connected to feeder one (57kW/phase, equally spread) and during the low load (16kW/phase) they will significantly change the voltage profile for the POCs connected to the feeder with DGs, see Fig. 2. The minimum loading value is based on measurements taken in several MV/LV substations and the load is spread equally between the feeders.

Without voltage control, the voltage profile for the all POCs will change and the POCs connected at the feeder with DGs will suffer from overvoltage, as shown in Fig. 2, where, the red dashed lines represent the voltage limits of the supply voltage $\left( \pm 10 \% U_{n}\right)$, (discussed in [7], [8]). The customers connected beyond the POC-10 in feeder 1 will suffer from higher voltage, not complying with the standard for supply voltage. But this is only one of the possible configurations (not the worst case), which will be further explored.

\section{EXAMINED GRID SITUATION}

The examined grid model was based on a typical situation in the Dutch LV network. The modeled grid consists on a typical MV/LV substation (with a 400kVA transformer) supplying $240 \mathrm{POCs}$, which are equally distributed among four feeders. This results in 60 customers on each feeder and 20 connection points for each phase. The loading of the grid represents a typical loading of a grid of this size and is based on measurements in several $\mathrm{MV} / \mathrm{LV}$ substations in the Netherlands. In the model, each feeder is protected by $250 \mathrm{~A}$ fuses, limiting the load and generation connected via this feeder, see Fig. 1 and Fig. 4.

The future grid will be challenged by the connection of a considerable amount of intermittent DGs supplying the grid. For instance, high penetration of photovoltaic generators, connected to the LV grid, will represent a considerable fluctuating generation. Therefore, a sudden increase or a sudden decrease of the generation will affect the voltage profile for all customers connected to the grid almost simultaneously. On the one hand, the future grid should be able to handle these fluctuations and provide customers with a high continuity of supplied electricity. On the other hand, the future grid should also be able to accommodate the considerable amount of DG without degradation of the voltage levels for the customers connected.

Therefore, the examined voltage control strategies are applied for the LV grid configuration with a maximum allowable amount (due to the grid's constraints) of DG dispatched to three of the feeders. In the examined grid simulations is always considered one feeder without DG. This should represent the scenario, where the DGs are dispatched in other feeders resulting in the voltage rise in these feeders. And at the same moment, the feeder without DG will be affected only by the voltage drop due to its loading without DG. Hence, the voltage difference between these feeders will be the highest one. The network's constraints are taken into account, limiting the generation dispatched. In accordance with the grid's constraints, the amount and the place of the generation from DGs was estimated with the aim to present the worst combination from the voltage level point of view.

Table I provides the locations and connections of the DGs in the examined LV grid situation.

\section{TABLE I. CONNECTED DG TO THE EXAMINED LV GRID}

\begin{tabular}{|c|c|c|}
\hline Feeder & $\begin{array}{c}\text { POCs with } \\
\text { DG }\end{array}$ & $\begin{array}{c}\text { Peak } \\
\text { generation } \\
\text { per feeder }\end{array}$ \\
\hline 1 & $1 . .20$ & $57 \mathrm{~kW} /$ phase \\
\hline 2 & $17 . .20$ & $57 \mathrm{~kW} /$ phase \\
\hline 3 & $17 . .20$ & $57 \mathrm{~kW} /$ phase \\
\hline 4 & none & none \\
\hline
\end{tabular}

The total load profile of the LV grid, the generation profile of the DGs (photovoltaic generators) and only the consumption component of the examined LV grid without generation are depicted in Fig. 3. The described profiles are based on real measurements in the MV/LV substations and on output of PV generators.

\section{SMART TRANSFORMER}

$\mathrm{MV} / \mathrm{LV}$ transformers used today have a manual tap changer (off-line change). The tap position is usually set to offset the expected voltage drop among the LV feeder and the voltage drop on the transformer during high load. 


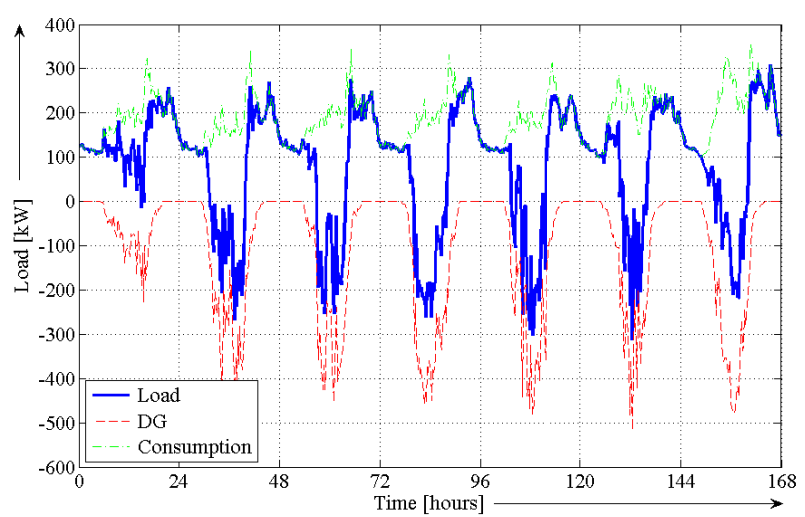

Fig. 3. Total load profile of examined LV grid, where the generation component from the DGs and only the consumption component are depicted separately.

Therefore, the concept of an intelligent substation, equipped with intelligent controllable transformer aims to attenuate the concerns about voltage levels in the grid with high penetration of DG.

The smart transformer is in its nature a $\mathrm{MV} / \mathrm{LV}$ transformer which is equipped with an electronically operating tap changer. This allows changing its tap position online with small voltage steps and in a broad range of voltages in order to reach the desired value of the LV side voltage given by the control mechanism. Therefore, the voltage at the LV side is no longer directly linked to the loading of the MV grid [6].

\section{CONTRol Strategies For The SMART TRANSFORMER}

The smart transformer will be able to control the voltage of its LV side. However, in order to allow the accommodation of DG in the future grid, the connection of DG should not be restricted or limited by its placement in the grid. The dispatch of the DG connected will lead to a rise in voltage. This will depend on the grid's impedance to the POC with connected DG. Therefore, the DGs connected at the end of the feeder will represent a worst case scenario for the voltage profile in the LV grid, resulting in significant voltage increase [4], [9].

Taking into account the DGs dispatched to the LV grid and the load profiles, different control strategies and their impact on the voltage levels for the customers connected has been investigated, as follows:

\section{A. Control of the bus bar voltage}

This control method is based on the assumption, that the bus bar voltage at the substation will keep constant value $\left(U_{n}=230 \mathrm{~V}\right)$. The main advantage of this control strategy is the implementation of all control measurements within the substation alone (point 1 in Fig.4.), diminishing the requirements on the communication infrastructure.

\section{$B$. Constant voltage on the end of the feeders}

In that case, the control of the transformer's tap position is based on the presumption, that the voltages at the end of all feeders (points 2) will be assessed. The tap position will be chosen in order to reach $U_{n}$ value on average among all the ends of feeders. This method

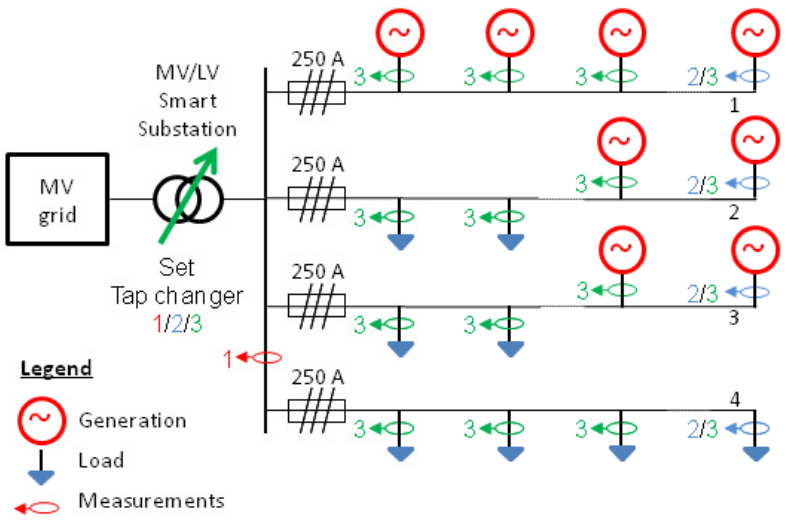

Fig. 4. LV grid equipped with smart transformer. The different control approaches of the transformer's tap position are schematically depicted.

requires a smart metering infrastructure (at least at the end of the feeders) in order to assess online current voltage levels and communicate them in real time to the substation.

\section{Assessing all voltages of all POCs connected}

The last examined control method assesses the transformer's tap position with regard to the measurements taken among all POCs connected in the LV grid (points 3). The aim is to keep all POCs voltages at desired values $U_{n}$ on average. The voltage levels at all POCs connected have to be assessed and communicated in real time to the substation in order to set the tap position of the smart transformer. In comparison to the previous mentioned strategies, this method has the highest requirements on the communication infrastructure and on the smart meters.

The model of examined LV grid equipped with the smart transformer and depicted measuring points for tap position optimization is shown in Fig. 4.

\section{VOLTAGE CONTROL RESULTS}

In accordance to the examined LV grid configuration, the voltage profiles for all strategies (mentioned in section V.) have been carried out. The results are represented in box plot figures for each method. Each box in figure represents the voltage levels for specified POC. The central marks of the boxes are the median values, the edges of the box are the $25^{\text {th }}$ and $75^{\text {th }}$ percentiles and the outliers are plotted individually. In accordance to the final draft of the revised EN50160 standard for the supply voltage, the voltage limits $\left(U_{n} \pm 10 \%\right)$ are plotted in figures, as well [8].

Moreover, in order to compare the results with the current situation, the voltage profiles for the examined configuration of DGs dispatched to the grid without smart transformer have been assessed, see Fig. 5. According to the results presented, the voltage profiles in the current grid situation without the smart transformer, will not comply with the standard for the supply voltage. The customers connected at the feeders, where the generations take place will suffer from overvoltage (from the POC 


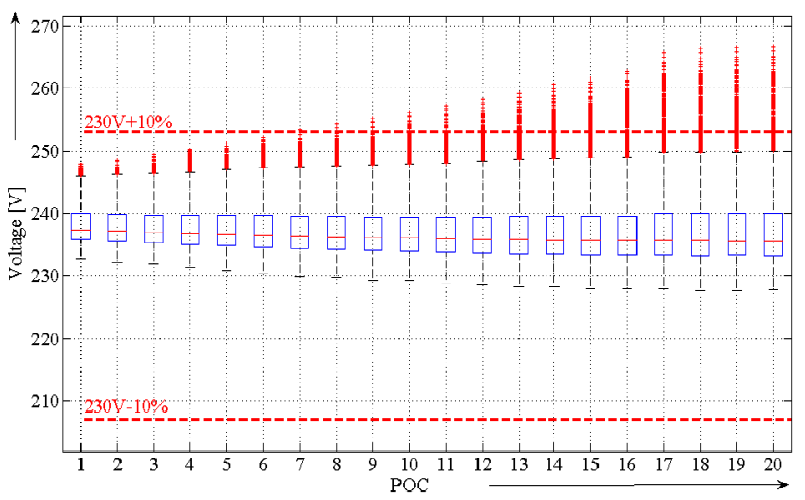

Fig. 5. Voltage profile of examined LV grid for each POC, the situation without smart transformer.

number 7 on, see Fig. 5). The higher voltages for all POCs will be due to the fixed tap position of the transformer, which has to be chosen, in order to offset the voltage drop among the LV grid during high load (also without DG). The connection of DGs will lead to a decrease of transformer loading, lower voltage drop on transformer and consequently to higher voltages in the LV grid (compared to the situation without DGs), see Fig. 5.

\section{A. The bus bar voltage stays constant}

In accordance to this voltage control method, the smart transformer will adjust its tap position at every moment, in order to keep the voltage at the bus bar steady. As a result of this control method, the voltage levels for each POC are depicted in Fig. 6. With this control strategy, the voltage levels at all POCs will comply with the standard for supply voltage. Near the transformer, the voltages tend to be the steadiest near the desired value. However, even the voltages at the end of the feeder, where the generation take place, are within the limits $\left(U_{n} \pm 10 \%\right)$, see the outliers in Fig. 6 at the POCs at the end of the feeder.

\section{$B$. The voltage at the end of the feeder stays constant}

In accordance to this voltage control method, the smart transformer will adjust its tap position in order to keep the average voltage at the end of the feeders constant. The voltage levels for each POC are depicted in Fig. 7. All the

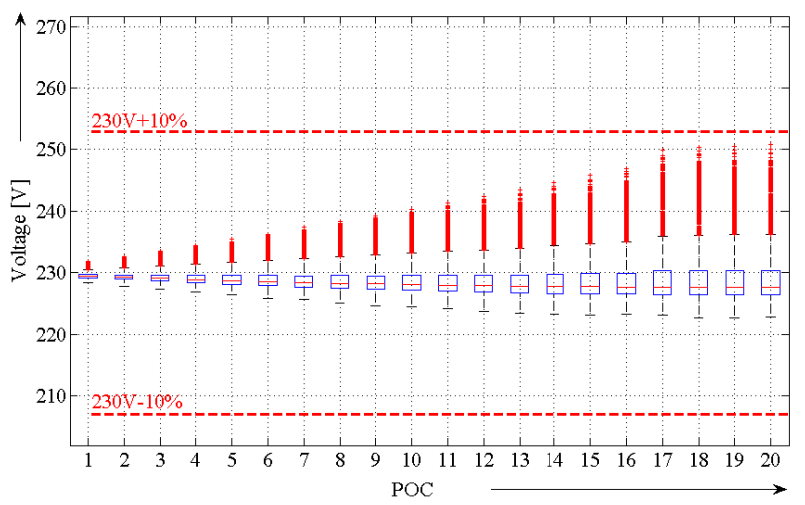

Fig. 6. Voltage profile of examined LV grid for each POC, the tap position is adjusted in order to keep the bus bar voltage steady $(\operatorname{method} \mathrm{A})$.

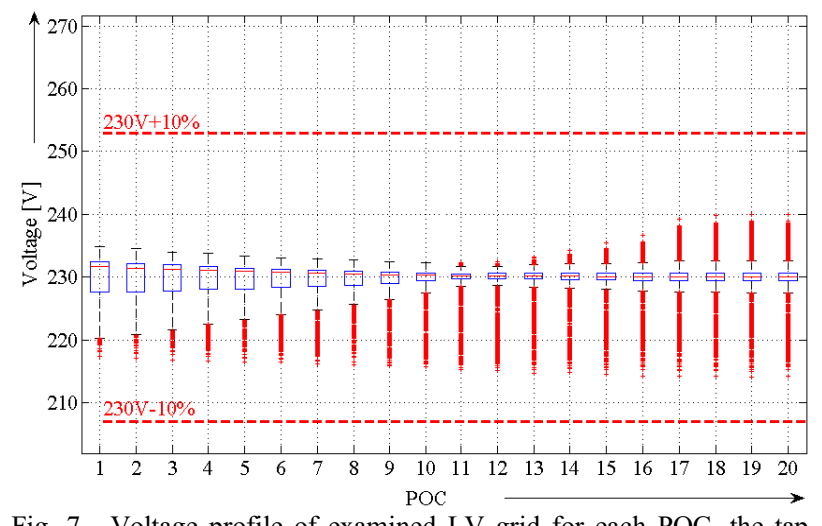

Fig. 7. Voltage profile of examined LV grid for each POC, the tap position is adjusted in order to keep the average voltages at the end of the feeders on desired value (method B).

levels of voltage for all POCs connected are within the limits given by the standard for supply voltage EN50160 [8]. The control adjustment is focusing on the voltages at the end of the feeder. However, due to the inhomogeneous generation in the feeders, there is a higher voltage spread among the feeder at their ends. This corresponds to the voltage differences between the feeders, where the generation take place and the feeder without generation, see Fig. 7.

\section{Assessing all voltages of all POCs connected}

The last examined control method assesses the voltage profiles of all POCs connected, in accordance to the proposed control approach. The goal of the control method is to keep all POCs connected with the desired voltage on average $\left(U_{n}\right)$. The estimated results are shown in Fig. 8. All voltages at all POCs connected are complying with the standard for supply voltage EN50160 [8]. Moreover, all voltages are within the limits $\left(U_{n} \pm 10 \%\right)$. The majority of the values (see $25^{\text {th }}$ and $75^{\text {th }}$ percentile in Fig. 8) have only a small voltage deviation from the $U_{n}$ value. Therefore, the customers connected will experience only relatively small deviations of their voltages from $U_{n}$.

However, the real time assessment of the voltages among the whole LV grid will be very demanding on the data infrastructure (the worst among the proposed control strategies).

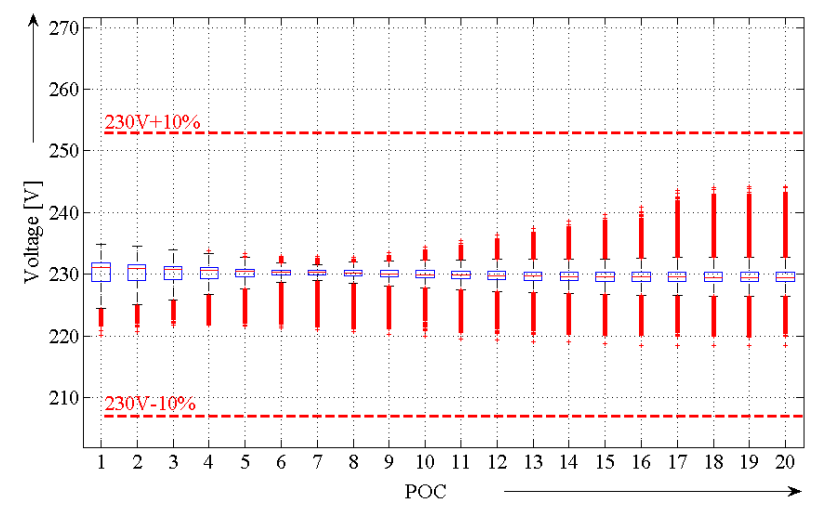

Fig. 8. Voltage profile of examined LV grid for each POC, the tap position is adjusted in order to keep the average voltage of all POCs connected on desired value (method C). 


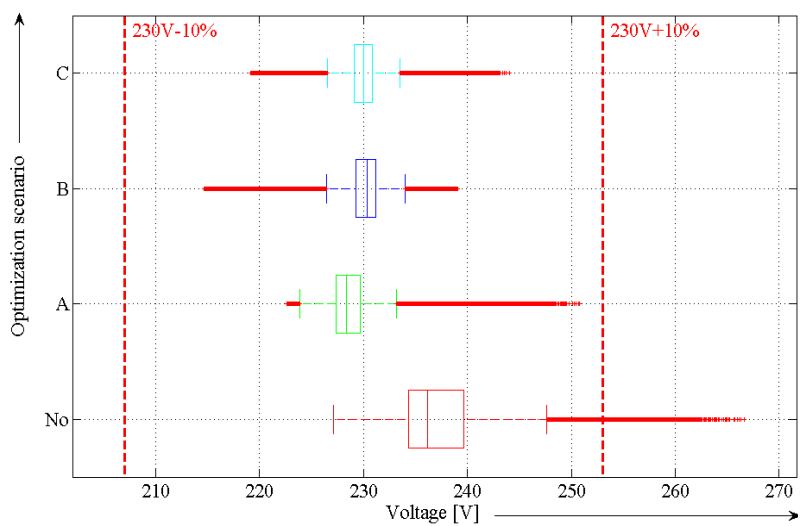

Fig. 9. Voltage profiles of examined LV grid for each control method, the tap position adjusted in accordance to the methods $A, B$ and $C$. The voltage profile without smart transformer is shown as method No.

\section{RECOMMENDATION FOR IMPLEMENTATION}

The examined strategies have shown the impact of the smart transformer on the future grid. Without the smart transformer, the decentralized generators are limited in the dispatch of their energy. The location (the grid's impedance) from the substation plays a fundamental role in voltage rise among the LV grid without the voltage control. The voltages in this case will not comply with the supply voltage standard; see Fig. 5 and Fig. 9. This will be reflected on the technically feasible amount of DG accommodated in such a kind of LV grid. In this case, the DG will be restricted by their size and their location in the LV grid.

The examined voltage controls of the smart transformer have shown significant improvements in the voltage levels for all POCs connected. In the LV grid with the smart transformer, the voltages at all POCs will be complying with the supply voltage standards for all examined methods with the smart transformer.

The different voltage profiles for the POCs are reflecting the control strategies for the transformers tap position. Among all POCs, the differences in voltage control approaches are not significant. The mean values and the standard deviation of the voltages for all POCs for are presented in the Table II.

TABLE II. STANDARD DEVIATIONS AND MEAN VALUES OF THE VOLTAGES FOR DIFFERENT CONTROL STRATEGIES

\begin{tabular}{|c|c|c|}
\hline $\begin{array}{c}\text { Control } \\
\text { strategy }\end{array}$ & $\begin{array}{c}\text { Standard } \\
\text { deviation [V] }\end{array}$ & $\begin{array}{c}\text { Mean value } \\
\text { [V] }\end{array}$ \\
\hline $\begin{array}{c}\text { Without } \\
\text { Control }\end{array}$ & 5.7 & 237.9 \\
\hline $\begin{array}{c}\text { At } \\
\text { bus bar (A) }\end{array}$ & 3.6 & 229.4 \\
\hline $\begin{array}{c}\text { At } \\
\text { the end of the } \\
\text { feeders (B) }\end{array}$ & 3.0 & 229.7 \\
\hline $\begin{array}{c}\text { At } \\
\text { all POCs on } \\
\text { average (C) }\end{array}$ & 2.7 & 230.0 \\
\hline \multicolumn{2}{|c|}{} \\
\hline
\end{tabular}

The voltage profiles over all POCs for each control method and for the strategies without the smart transformer (method No) are depicted in Fig. 9. The median values and the $25^{\text {th }}$ and $75^{\text {th }}$ percentiles are in

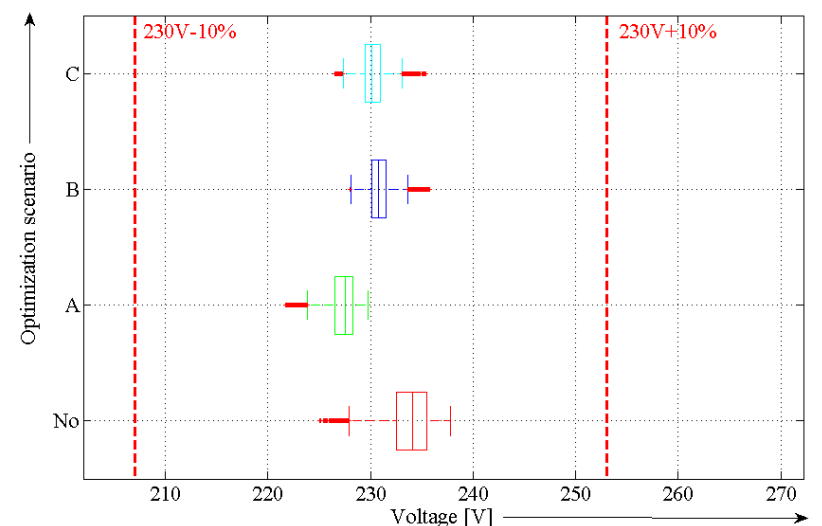

Fig. 10. Voltage profiles of examined LV grid configuration without DG, for each control scenario, the tap position adjusted in accordance to the scenarios $A, B$ and $C$. The voltage profile without smart transformer is shown as scenario No.

relatively small intervals. On one hand, as depicted in Fig. 9, the highest voltage values (with the smart transformers) for outliers are for the method A. However, it has to be pointed out; these outliers are the voltage values at the end of the feeders due to the concentrated generation in the end of the feeders, see Table I. A similar situation represents the worst case scenario and will be nearly never the real grid situation. On the other hand, the method A represents the easiest method for implementation, where the requirements on the smart metering infrastructure and on the data processing from the SM are diminished. The real time measurements for assessing the tap position will take place within the substation alone. Therefore, the substation implementation will be significantly simplified. With this control approach, the substation equipped with the smart transformer might be also used for current grids without SM infrastructure as a modular solution.

The estimated voltages for the same voltage control method without DG was assessed as well, see Fig. 10. Where only the load component of the load, see Fig. 3, is taken into account. Inter alia the presence of load component alone will decrease voltage spread for each POC, because the voltages along all the feeders in examined grid situation without DG are only decreasing.

\section{CONCLUSIONS}

This paper addresses the importance of the voltage control for the future grids. Where high penetration of distributed generators, dispatched to the distribution network, might cause unwanted voltage rises for the customers connected to this grid. Moreover, the voltage fluctuations in the medium voltage grid might be amplifying this effect in subsequent low voltage grids. Due to the different mixture of generators in the future grid, the voltages for the customers might be easily driven out of the limits forced by supply voltage standards.

The concept of smart transformer, as a part of the smart substation in future grid, is presented in order to attenuate this unwanted effect on the voltage profile for the connected customers. In comparison to the current grid situation, the implementation of the smart 
transformer will significantly improve the voltage profiles for all customers connected.

The simulations point out, that the standard low voltage grid equipped with the smart transformer will be able to accommodate the distributed generators (up to the technical limits given by the grid's constraints), regardless to their location among the feeder. And the voltage profile of the customers will still comply with the supply voltage standards. Therefore, a future grid will be more flexible in regard to the accommodation of the distributed generators.

In addition, the implementation of the smart transformer will attenuate the fluctuations of the medium voltage grid and contribute to the improvements of the supplied voltage quality for the customers.

This paper presents the control strategies of the smart transformer and takes into account the requirements of the smart metering infrastructure. The proposed solution will assure the voltage profile complying with the supply voltage standards in the future grid. Moreover, the implementation will be feasible also for present day grids without smart metering infrastructure.

\section{REFERENCES}

[1] CEER, "Energy regulators' pledge to ensuring good quality of electricity supply," FS-0901, 2009.

[2] P. Kadurek, J.F. Cobben, and W.L. Kling, "Additional features of smart metering," Young Researchers Symposium 2010, 2010, pp. 1-6.

[3] J. Cobben, W. Kling, and J. Myrzik, "Power quality aspects of a future micro grid," 2005 International Conference on Future Power Systems, 2005, pp. 5.

[4] Cobben J. F. G., "Power Quality Implications at the Point of Connection," $\mathrm{PhD}$ Thesis, Eindhoven University of Technology, 2007.

[5] S. Bhattacharyya, Z. Wang, J. Cobben, J. Myrzik, and W. Kling, "Analysis of Power Quality Performance of The Dutch Medium And Low Voltage Grids," ICHQP 2008 13th International Conference on Harmonics and Quality of Power, 2008, p. 1-6.

[6] P. Bauer and S.W. de Haan, "Electronic tap changer for $500 \mathrm{kVA} / 10 \mathrm{kV}$ distribution transformers: design, experimental results and impact in distribution networks," Industry Applications Conference, 1998, pp. 1530-1537 vol.2.

[7] ERGEG, "Towards Voltage Quality Regulation in Europe An ERGEG Conclusions Paper," E07-EQS-15-03, 2007.

[8] CENELEC, "EN 50160 - Voltage characteristics of electricity supplied by public distribution networks," Final draft EN50160:2009E, 2009.

[9] A. Baggini, "Handbook of power quality," 2008. 